\title{
LE CRU, LE CUIT ET LE POURRI DANS LE VICE-CONSUL DE MARGUERITE DURAS
}

Hélène Caron, Université de Toronto

\section{Résumé}

Bien que plusieurs articles critiques aient déjà avancé de nouvelles perspectives sur le texte $L e$ Vice-Consul (LVC) de Marguerite Duras, l'analyse de la représentation de la nourriture dans ce roman n'a fait l'objet d'aucune étude. Cet article tente donc de combler cette lacune en proposant d'analyser les images du cru et du cuit, images qui se rejoignent sous les notions du pourri et de l'abject, tout en offrant quelques interprétations de l'un des passages critiques du roman où la folle mendiante croque la tête d'un poisson vivant.

Quarante ans après sa date de publication, LVC de Marguerite Duras continue à troubler et à perturber les lecteurs et lectrices, entre autres à cause de certaines descriptions abjectes qui fascinent et repoussent tout à la fois :

Elle reprend le poisson et, lui montrant, elle croque la tête en riant davantage encore. Le poisson guillotiné remue dans sa main. Elle doit s'amuser de faire peur, de donner la nausée. (205) [...] La bouche pleine du poisson cru, elle chante. (206)

Qui n'a pas été choqué, en effet, en lisant ce passage où la mendiante folle croque la tête d'un poisson vivant devant un Européen pétrifié par la peur ? La répugnance et l'horreur que ce passage a suscitées chez moi m’ont en fait poussée à étudier le langage de la nourriture, et plus précisément celui du cru et du cuit qui se présente sous la forme d'une opposition, l'une des nombreuses oppositions dans le roman. Le langage de la nourriture peut-il offrir ici une clé à la 
compréhension et à l'interprétation du texte ? Et surtout, quelles significations recèle le passage de la décapitation du poisson vivant ? Certains critiques, tels James Brown et Gian-Paolo Biasin, ont utilisé soit une approche sémiotique, soit une approche plus ethnologique basée sur les fonctions de la nourriture ${ }^{1}$ pour découvrir et expliquer les sens cachés du signe culinaire dans les textes littéraires. Tout en incorporant les méthodes de ces deux approches, je me concentrerai davantage sur l'aspect anthropologique de la nourriture, tel que présenté par Claude Lévi-Strauss dans son livre Le cru et le cuit, et plus particulièrement sur le rapprochement qu'il a relevé entre le cru et la nature, et le cuit et la culture :

La conjonction d'un membre du groupe social AVEC la nature doit être médiatisée par l'intervention du feu de cuisine, à qui revient normalement la charge de médiatiser la conjonction du produit cru et du consommateur humain, et DONC par l'opération duquel un être naturel est, tout à la fois, cuit et socialisé. (342, mon emphase en caractères majuscules)

De plus, puisque le langage de la nourriture peut être étudié sous plusieurs angles ${ }^{2}$, nous aborderons l'analyse de toute nourriture, solide et liquide, ingérée ou rejetée par la bouche des personnages.

\section{a. Le cru}

La mendiante aura parcouru beaucoup de chemin avant de parvenir au moment culminant de la scène du poisson cru. À la maison, bien que maltraitée par sa mère, la jeune fille avait toujours un bol de riz chaud à se mettre sous la dent. À l'adolescence, elle se voit chassée du foyer familial par sa mère suite à un viol qui la laisse enceinte ${ }^{3}$. Bien qu'au tout début ce soit la mère qui rejette sa fille, par la suite la fille se décide à rejeter sa mère ; selon les mythes relevés par Lévi-Strauss, le rejet de la mère par une fille équivaut à un rejet de la culture et implique 
ainsi un mouvement vers la nature. La jeune mendiante quitte ainsi définitivement le monde de la culture pour celui de la nature.

Dans la lumière bouillante et pâle, l'enfant encore dans le ventre, elle s'éloigne, sans crainte. Sa route, elle est sûre, est celle de l'abandon définitif de sa mère. Ses yeux pleurent, mais elle, elle chante à tue-tête un chant enfantin de Battambang. (LVC 28)

Le rejet de la maternité et de la culture par la mendiante est mis en lumière à maints niveaux par le biais de la nourriture : la mère de la mendiante la menace d'empoisonner son riz chaud si cette dernière revient à la maison après en avoir été chassée ; la mendiante «s'efforce de vomir l'enfant, de se l'extirper, mais c'est de l'eau de mangue acide qui vient »(18), et enfin, l'enfant qu'elle porte la mange aussi :

...nuit et jour l'enfant continue à la manger, elle écoute et entend le grignotement incessant dans le ventre qu'il décharne, il lui a mangé les cuisses, les bras, les joues. (18)

Abandonnée par tous, la mendiante mange ce que la nature peut lui fournir, soit du riz vert, des oiseaux morts et des fruits non mûrs comme des bananes et des mangues.

The fact that Peter Morgan (le narrateur) has the young girl eat roots, green fruits and dead birds is not an insignificant detail. This behavior connotes marginality by showing how the girl, expelled from her family, abandons the culinary code of normal and integrated life and becomes half-animal and half-human. (Sherzer, Discourse 372)

La nouvelle alimentation de la jeune fille marque effectivement son passage à la marginalité et à l'altérité par rapport à son propre groupe de référence. Cependant, je suis en 
désaccord avec Sherzer lorsqu'elle affirme que la jeune fille devient mi-animal, mi-humain ; cette transformation s'effectue plus tard dans le récit, et cet aspect sera discuté plus loin. Notons simplement ici que la jeune fille s'éloigne de ce qui est considéré «culture » ou « civilisation » en mangeant des aliments crus et non mûrs, et cette marginalisation ou position d'altérité ne représente que le premier pas dans son cheminement vers la déchéance. ${ }^{4}$

Les mangues qu'ingère la mendiante possèdent une signification féminine et érotique, tel que le souligne Eve Jackson : «Fruit similes are common in erotic descriptions of the female body, smooth and curvaceous, rounded and firm or fleshy, juicy; breasts like peaches, mangos... » (42) Mais les mangues consommées n'étant pas mûres, elles perdent à la fois leur valeur nutritive et leur signification érotique, ce qui est reflété par la maigreur de la jeune fille. La faim devient rapidement une obsession - mentionnée au moins quatorze fois dans le texte pour décrire l'état physique de la mendiante -, la jeune fille devant non seulement pourvoir à ses propres besoins mais aussi à ceux de son foetus, «L'enfant mange tout, riz vert et mangues. » (15) Cette faim qui la dévore la pousse à se prostituer et à mendier ; ainsi, après avoir été rejetée par sa mère, le tourbillon qui emporte la mendiante de la culture à la nature l'emporte encore plus loin, c'est-à-dire vers un début de folie.

...elle met les dents dans le poisson, le sel croque avec la poussière. [...] La salive monte, jaillit dans la bouche, c'est salé, elle pleure, elle bave, elle n'a plus eu de sel depuis longtemps, c'est trop, c'est beaucoup trop, elle tombe et, tombée, elle continue à manger la nourriture. (LVC 21-22)

Quelques lignes plus loin, elle avale une poignée de poussière croyant manger du riz chaud. Le domaine du cuit-culture n'est plus que rêve et hallucination - de même que celui du cru-nature puisqu'elle se retrouve dans une espèce de non-lieu entre nature et folie. Sa déchéance 
est entamée, elle n'a plus le moyen de retourner à la nature, et encore moins de retourner à la culture. Ceci se reflète d'ailleurs dans les «nouveaux » aliments qu'elle commence à ingérer : ...elles (les mendiantes) voient des morceaux de cochon étincelants sur des étals (de marchés publics), des nuées de mouches bleues regardent avec elles, plus près. Aux vieilles femmes ou aux vendeurs de soupe, elle demande chaque fois un bol de riz. Elle demande des choses différentes, riz, os de cochon, poisson, vieux poisson quoi. Qu'est-ce que ça peut vous faire de me donner un vieux poisson? Parce qu'elle est si jeune, quelquefois on lui donne. Mais la règle, c'est le refus. (17)

Dans ce passage où l'on donne un poisson pourri à la mendiante, quatre aspects importants du roman sont soulevés. Premièrement, la fonction référentielle réaliste de la nourriture $^{5}$ permet au lecteur non seulement de situer la protagoniste dans son contexte cambodgien, mais aussi de compatir avec la difficulté que la jeune fille répudiée et enceinte rencontre pour se nourrir. Deuxièmement, selon une optique anthropologique et sociologique, la disponibilité de la nourriture n'est pas un problème à l'intérieur du groupe de référence originel de la mendiante, mais la répartition des vivres est réservée seulement à ceux qui en ont les moyens ; l'opposition pauvreté / richesse commence ainsi à être abordée dans le texte. Troisièmement, le fait que la plupart de ses compatriotes lui refusent de la nourriture, sauf deux ou trois femmes, démontre le refus d'une société de venir en aide à ses plus démunis, soit une jeune mendiante enceinte. Enfin, le thème de la pourriture qui est l'aboutissement du «cru périmé » et qui, selon les mythes relevés par Lévi-Strauss représente la décomposition de l'être et l'approche de la mort $(299,344)$, est aussi abordé par la mention du « vieux poisson » dans le 
passage ci-dessus. Ce vieux poisson, qui commence à sentir mauvais et à pourrir, prépare le lecteur à ce qui s'ensuivra de l'âme et du pied blessé de la mendiante.

Une fois né, le bébé, une fille, rejette la mère à son tour en refusant le lait maternel et tout autre nourriture : «D’une jonque on a donné du riz chaud, elle a mâché le riz longuement et de bouche à bouche le lui a reversé, l'enfant a vomi. » (54) La mère rejette l'enfant, l'enfant rejette la mère — relation mère-fille créée à deux reprises mais vouée à l'échec, ainsi le rejet de la culture demeure ${ }^{6}$. La mendiante, après avoir donné son enfant à une femme blanche, sombrera définitivement dans la folie et la déchéance, outrepassant la limite du domaine de la nature. Elle sera ainsi passée du cuit / culture au cru / nature par le fait d'être rejetée par sa mère - et par son propre rejet de sa mère par la suite —, puis du cru / nature, à la déchéance / pourriture, à cause de la prostitution, de la mendicité et de la désintégration émotionnelle et physique qu'elle endure. En fait, l'ingestion de vieux poisson marque le début pour elle de la consommation d'aliments abjects: déchets de table des autres servis après la fête à l'ambassade, déchets de table trouvés lors de la fouille des poubelles de Calcutta, poisson cru et vivant fraîchement pêché.

Lorsque la nourriture apparaît comme objet polluant, elle ne l'est comme objet oral que dans la mesure où l'oralité signifie une frontière du corps propre. Une nourriture ne devient abjecte que d'être un bord entre deux entités ou territoires distincts. Frontière entre la nature et la culture, entre l'humain et le non-humain. (Kristeva 90)

Si l'identité de la jeune fille est un reflet de ce qu'elle ingère comme la maxime de Brillat-Savarin le prétend ${ }^{7}$, sa déchéance est alors entamée. L'ingestion d'aliments pourris ou rejetés par les autres se reflète au niveau de son âme qui elle aussi se désintègre, ce qui crée une redondance narrative, la description acquérant une fonction narrative. «Les restes sont des 
reliquats de quelque chose mais surtout de quelqu'un. Ils polluent $\mathrm{du}$ fait de cette incomplétude. » (Kristeva 91) Même si le corps de la mendiante continue de vivre, l'essence qui faisait d'elle une personne est morte, la perte complète de ses cheveux - autre exemple de redondance - en faisant état. L'ingestion d'aliments souillés ${ }^{8}$ se reflète par une autre manifestation corporelle externe pour le moins répugnante : le pied infecté, exhalant une odeur immonde et grouillant de vers, accompagnera la mendiante à partir de son accouchement jusqu'à la fin du récit. La perte des cheveux, le pied pourri, l'ingestion de restes de table et d'aliments pourris marquent tous la déshumanisation du personnage de la mendiante (Carruggi 122-123), et le début de son cheminement vers un état de pourriture, d'abjection.

Un seul mot est prononcé et répété par elle à la fin du récit, soit « Battambang », l'endroit d'où elle vient. Par ailleurs, elle chante continuellement la même comptine de son enfance « qui dit que le buffle mangera l'herbe mais qu'à son tour l'herbe mangera le buffle lorsque l'heure sonnera » (58), paroles qui rappellent les relations destructrices mère-fille, culture-nature qu'elle a vécues. La transformation de la mendiante qui passe de l'état de culture à celui de nature est complète lorsqu'elle acquiert à la fin une apparence mi-humaine, mi-animale :

Elle lui tourne le dos, elle va droit vers la lagune et y pénètre, très, très prudemment, tout entière. La tête seule émerge à fleur d'eau, et très exactement comme un buffle ${ }^{9}$, elle se met à nager avec une hallucinante lenteur. (LVC 207)

\section{b. Le cuit}

À Calcutta, où la mendiante cambodgienne aboutit après des années de marche, les restants de table abondent grâce à la présence d'un cercle d'Européens, chargés de maintenir des relations diplomatiques. Ainsi, le personnage d'Anne-Marie Stretter, femme de l'ambassadeur, paraît comme une autre protagoniste dans ce roman. Si on accepte l'association de la mendiante 
avec le terme «cru», Anne-Marie et les autres Européens seraient donc représentés par le « cuit », c'est-à-dire la culture. Le contraste de ces deux mondes apparaît lorsque Anne-Marie Stretter ordonne aux serviteurs de mettre une bassine d'eau fraîche à la disposition des mendiants et des lépreux qui pullulent autour de sa résidence clôturée et surveillée. De plus, après la réception à l'ambassade française, Anne-Marie Stretter fait distribuer les restes aux démunis. Tandis que les gens meurent de faim autour d'eux, le groupe d'Européens fête en buvant du champagne et en mangeant des sandwichs au foie $\operatorname{gras}^{10} ;$ l'abondance du cuit règne.

...we read page after page of the utter sordidness and squalor in which hordes of lepers and beggars live wallowing all day long in the filth, stench and foulness of the Calcutta dumps alongside the stray dogs in total oblivion of their surroundings. Standing in stark contrast to this subhuman, alienating and animallike condition, is the glittering and dazzling glamor of the highly sophisticated Embassy society [...] where grapes, champagne, and foie gras are flown from the Metropolis to grace the table of the European hosts. (Ha 306-307)

Ces aliments, relevant du domaine du cuit puisque transformés, mettent en évidence les oppositions manque / abondance et pauvreté / richesse mentionnées plus tôt, mais établissent aussi une distance culturelle entre Calcutta et le cercle fermé auquel appartiennent les Européens. La nourriture ingérée lors de la réception et les propos de Ha marquent de plus la situation historique coloniale dans laquelle évoluent les protagonistes. D'habitude, en Occident, la consommation de ces aliments coïncide avec une célébration qui marque un événement spécial, dénotant fête, joie de vivre, richesse et surtout appartenance à un groupe de référence, aspects sémiotiques qui tranchent encore plus avec la toile de fond indienne et qui perdent toute signification lorsque les restes ne servent qu'à assouvir les besoins corporels des mendiants. 
Cependant, les invités dans LVC ne sont pas dupes: même en tentant de recréer une pseudoculture européenne grâce aux odeurs, aux textures et aux goûts des aliments du pays d'origine avec le foie gras et le champagne, la réalité est tout autre à l'extérieur des murs de la résidence de l'ambassadeur de France. Les apparences et les illusions de culture sont remises en question lorsque la plupart des invités boivent à outrance ; le champagne, tout comme le cognac et le whisky aussi mentionnés dans le récit, n’est donc rien de moins qu'une façon d'échapper à la dure réalité de la pauvreté de Calcutta, l'ivresse devenant un signe d'exutoire. S'agirait-il ici de la représentation d'une forme de culpabilité par rapport à l'arrogance coloniale, ou peut-être même d'une nostalgie pour une culture européenne idéalisée ? L’ivresse des personnages européens, qui traduit un malaise dû à un noeud d'émotions complexes tels le désenchantement, la tristesse, la folie et la mauvaise intégration au contexte indien, semble indiquer un certain rejet de la culture européenne. Ha abonde aussi dans ce sens, soulignant que même les barrières physiques ne peuvent isoler les Européens de l'environnement extérieur - l'Inde et ses habitants - ce qui se reflète dans la santé mentale et physique des Européens. (Ha 310-311) La folie est incarnée par le vice-consul, qui a tiré sur des lépreux à l'extérieur de sa résidence et qui, tout au long du roman, ne semble rien manger et s'enivre constamment.

Il y a, dans l'abjection, une de ces violentes et obscures révoltes de l'être contre ce qui le menace et qui lui paraît venir d'un dehors ou d'un dedans exorbitant, jeté à côté $d u$ possible, du tolérable, du pensable. C'est là, tout près mais inassimilable. Ça sollicite, inquiète, fascine le désir qui pourtant ne se laisse pas séduire. Apeuré, il se détourne. Écoeuré, il rejette. Un absolu le protège de l'opprobre, il en est fier, il y tient. Mais en même temps, quand même, cet élan, ce spasme, ce saut, est attiré vers un ailleurs aussi tentant que condamné. 
Inlassablement, comme un boomerang indomptable, un pôle d'appel et de répulsion met celui qui en est habité littéralement hors de lui. (Kristeva 9) ${ }^{11}$

Le vice-consul a éventuellement craqué sous le poids de toute l'abjection qui l'entourait, c'est-à-dire de la mendicité indienne à sa porte. Son état se rapproche ainsi, d'une certaine façon, de celui de la mendiante, en cela qu'il se rapproche de l'abject et de la pourriture ; le vice-consul n'a aucun ami et est évité par tous après l'incident de la tuerie. Il semble n'attendre que le verdict du consul de France, un peu comme s'il attendait l'annonce de sa propre mort puisqu'il ne fait que se désintégrer (émotionnellement) encore plus à Calcutta, il ne fait que pourrir.

Le malaise de vivre à Calcutta est ressenti par tous les Européens qui y sont, et même le repas que l'écrivain anglais s'apprête à manger y fait référence : «Charles Rossett déplie sa serviette et mange du curry indien. Le curry est fort, toujours trop fort ici, Charles Rossett le mange comme s'il y était condamné. » (LVC 46) La nécessité d'adhérer aux préceptes de la culture européenne s'avère cruciale pour que les personnages conservent leur tranquillité d'esprit et ne se sentent pas «condamnés » par leur environnement. Par exemple, pour échapper au mal de vivre de Calcutta, Anne-Marie et quelques hommes s'embarquent pour une île où ils sont entourés de luxe et d'opulence typiquement européens à l'hôtel Prince of Wales, luxe annoncé par le nom d'origine aristocratique anglaise. L'alcool continue là aussi de couler à flots. Et pour échapper à la chaleur de l'Inde, Anne-Marie Stretter compense avec un moyen européen : «Je vais chercher de la glace, celle-là est fondue, pendant la mousson elle fond si vite que... [...] Elle revient, tient la glace dans ses mains, se brûle, rit, la jette dans le seau à glace, sert du whisky. » (190) Celle qui boit beaucoup plus qu'elle ne mange persiste à croire aux rites européens, répétant les mêmes gestes inutiles pour se rafraîchir dans un contexte où le thé vert brûlant (LVC 47-48) est de rigueur pour combattre la chaleur : 
...Pendant la chaleur, oui, un conseil, il ne faut boire que du thé vert brûlant...

Seule cette boisson apaise la soif... se retenir de boire des boissons glacées... boire le thé vert, amer, râpeux, d'accord, mais on finit par aimer ça... c'est le secret de la mousson. (163)

Anne-Marie Stretter, malgré le fait qu'elle tend une main nourricière vers les mendiants de son quartier par l'entremise de son personnel, parvient à conserver sa tranquillité d'esprit en refusant de s'adapter à l'Inde et en maintenant ses distances grâce aux gestes répétés de culture européenne relevant du domaine du «cuit », pseudoculture plutôt, déstructurée au contact des cultures colonisées qu'elle prétend dominer.

L'analyse du cru dans LVC a mis en évidence le trajet de la déchéance de la mendiante. Le rejet de la jeune fille par sa mère tout d'abord, puis celui de la mère par la jeune fille, soustendent tous deux le cheminement de la mendiante du pôle de la culture à celui de la nature. Puis la faim, la mendicité et la prostitution l'ont menée à la folie, la rapprochant ainsi de la pourriture et de l'abject. L'analyse du cuit associé aux Européens, quant à elle, a mis en relief l'incongruité de la présence européenne en sol indien, provoquant l'ivresse des personnages et remettant en question la notion de culture dans un contexte autre. L'exemple du vice-consul qui boit beaucoup trop et dont l'état émotionnel se rapproche le plus de celui de la mendiante, nous montre que si l'extrême du cru périmé est la pourriture selon Lévi-Strauss, le cuit périmé peut lui aussi se rapprocher de cet extrême. Les deux méthodes utilisées pour faire ressortir le langage de la nourriture ont révélé les systèmes d'opposition mis en place par Duras, soit, évidemment, le cru et le cuit mais aussi la nature/la culture, la vie/la mort, la pauvreté/la richesse, le manque/l'abondance et les Européens/la Cambodgienne ${ }^{12}$. Malgré cette dernière opposition, la conjonction des trois personnages principaux, soit la mendiante, Anne-Marie Stretter et le vice- 
consul, se fait au niveau de leur maigreur (Carruggi 103-104), reflet de ce qu'ils ingèrent, mais aussi de leur folie et d'une pénurie de nature différente chez chacun. La maigre Anne-Marie, traitée mystérieusement pour les nerfs par le passé, se rapproche de la mort, qu'annoncent sa surconsommation d'alcool et son absence d'ingestion de nourriture solide; la fragilité adolescente du maigre vice-consul se voit à son état d'ébriété rapidement atteint lors de la réception ainsi qu'à sa folie ; la mendiante maigre pourrit lentement ${ }^{13}$ mais sûrement aux abords du Gange et ne profère toujours que les mêmes syllabes. Les manifestations extérieures de maigreur et de folie représentent des indices de leur déchéance commune, de leur mal de vivre et de leur position de victime de leur environnement. De plus, sous le signe du cru et du manque, la mendiante rejoint le vice consul placé sous le signe de la surconsommation du cuit et du distillé (l'alcool), les deux étant réunis dans la même déchéance abjecte de l'univers pourri du colonialisme.

Alors que pouvons-nous déduire de l'épisode du poisson cru mentionné en introduction? Quelles interprétations possibles pouvons-nous en tirer dans le cadre de notre présente analyse ? La première interprétation relève de la fonction réaliste : la mendiante étant folle et n'ayant rien d'autre à se mettre sous la dent, elle prend ce que la nature lui offre. Explication facile, certes, et qui mérite d'être suivie d'autres interprétations : peut-être Duras voulait-elle souligner avec ironie le choc du premier «dialogue », donc de la première rencontre entre les deux mondes opposés ? La mendiante, folle, mange du poisson dont la consommation est supposée contribuer à l'intelligence humaine, tandis que Charles Rossett est dégoûté par ce qu'il voit, habitué qu'il est à la nourriture cuite, préparée et assaisonnée, et à des manières de table d'où la décapitation in vivo et parler la bouche pleine sont exclus. «Le dégoût alimentaire est peut-être la forme la plus élémentaire et la plus archaïque de l'abjection. » (Kristeva 10) Il comprend alors 
l'impossibilité d'atteindre l'objectif qu'il s'était fixé en arrivant en Inde, soit de comprendre l'âme de ses habitants, puisqu'il ne pourra jamais franchir le fossé qui sépare son identité culturelle et économique de la mendicité indienne ; il est condamné à vivre dans la contradiction de la situation coloniale telle que soulignée par Memmi.

... a colonizer (term used by Memmi to refer to the colonial who does not accept colonization) is condemned to live in perpetual contradiction with her/himself. And no matter how genuine her/his sympathy for the colonized may be or how strong her/his rejection of the colonial power turns out to be, she/he still belongs to the oppressor group and enjoys privileges which accompany her/his position as the white master. (Ha 303)

Une autre interprétation de type sémiotique et anthropologique s'avère nécessaire pour éclairer davantage ce passage : le poisson, selon Chevalier, est entre autres «symbole de vie et de fécondité, en raison de sa prodigieuse faculté de reproduction et du nombre infini de ses oeufs. » (42) Le poisson est ainsi un signe de féminité, de maternité : en lui croquant la tête tandis qu'il est encore vivant, la mendiante illustre la déchirure qu'elle a vécue en tant que mère qui a dû abandonner son enfant, en tant que femme devenue stérile par la suite et, surtout, en tant qu'être humain tombé dans la déchéance, désertant le monde de la culture pour épouser de force celui de la nature. Si le poisson représente à présent la mère de la mendiante, sa décapitation représenterait le rejet de la mère par l'enfant mendiante. Et finalement, puisque la mendiante a tiré ce poisson vivant d'entre ses seins avant de le manger, il est aussi possible que le poisson représente son propre enfant: la décapitation du poisson cru signifierait le rejet de l'enfant par sa mère mendiante. Le buffle mange l'herbe, l'herbe mange le buffle, chante continuellement la 
mendiante. La mendiante rejette sa féminité et sa mère, la mendiante rejette son enfant.

\section{Bibliographie}

\section{$\underline{\text { Euvres citées }}$}

Brillat-Savarin. Physiologie du goût. (1ère éd. 1826). Paris : Hermann, 1975.

Carruggi, Noëlle. Marguerite Duras. Une expérience intérieure : “le gommage de l'être en faveur du tout”. New York : Peter Lang, 1995.

Chevalier, Jean et Alain Gheerbrant. Dictionnaire des symboles. Paris : Seghers, 1974.

Duras, Marguerite. Le Vice-Consul. Paris : Gallimard, 1966.

Ha, Marie-Paule. "Duras on the Margins." The Romanic Review 83, 3 (1993): 299-320.

Jackson, Eve. Food and Transformation : Imagery and Symbolism of Eating. Inner City, 1996.

Kristeva, Julia. Pouvoirs de l'horreur. Essai sur l'abjection. Paris : Seuil, 1980.

Le Petit Robert 1. Paris : Robert, 2004.

Lévi-Strauss, Claude. Le cru et le cuit. Paris : Plon, 1964.

Sherzer, Dina. "How Discourse Means : A View from Marguerite Duras's Le Vice-consul." Neophilologus 76, 3 (July 1992) : 370-382.

\section{$\underline{\text { Euvres consultées }}$}

Bal, Mieke. "Un roman dans le roman : encadrement ou enchâssement ? Quelques aspects du Vice-consul." Neophilologus 58 (1974) : 2-21.

Bakthine, Mikhaïl. L'œuvre de François Rabelais et la culture populaire au Moyen Âge et sous la Renaissance. Traduit du russe par Andrée Robel. Paris : Gallimard, 1970.

Biasin, Gian-Paolo. The Flavors of Modernity : Food and the Novel. New Jersey : Princeton UP, 1993.

Brasseur Wibaut, Tania. La gourmandise de Colette. Paris, Toronto : Harmattan, 2004. 
Brown, James W. "On the Semiogenesis of Fictional Meals.” Romanic Review 69 (1978) : 322335.

Cotoni, Marie-Hélène, Ed. Nourritures et écriture. Nice : U de Nice-Sophia Antipolis, 1999.

Counihan, Carole et Penny Van Esterik, Ed. Food and Culture : A Reader. New York : Routledge, 1997.

Douglas, Mary. Implicit Meanings. Selected Essays in Anthropology. $2^{\text {nd }}$ edition. London, New York : Routledge, 1999.

Gigante, Denise. Taste. A Literary History. New Haven / London: Yale UP, 2005.

Gilbert, Nina. "Duras' Le vice-consul and the Itinerant Discourse of Feminism." Canadian Review of Comparative Literature/Revue Canadienne de Littérature Comparée 21, 3 (Sept. 1994) : 311-324.

Goodman, Alan H., Darna L. Dufour et Gretel H. Pelto, Eds. Nutritional Anthropology : Biocultural Perspectives on Food and Nutrition. Toronto: Amyfield, 2000.

Hertz, Rachel. "Odor Memory : Review and Analysis." Psychonomic Bulletin \& Review 3.3 (1996) : 300-313.

Hitchcott, Nicki. "Comment cuisiner son mari à l'africaine : Calixthe Beyala's Recipes for Migrant Identity." French Cultural Studies 14, 2 [41] (June 2003) : 211-220.

Jeanneret, Michel. Des mets et des mots. Banquets et propos de table à la Renaissance. Paris : Corti, 1987.

Lévi-Strauss, Claude. L'origine des manières de table. Paris : Plon, 1968.

Lupton, Debrah. "Food, Memory and Meaning : the Symbolic and Social Nature of Food Events.” The Sociological Review 42, 4 (November 1994) : 664-685.

McIntosh, Alex Wm. Sociologies of Food and Nutrition. New York : Plenum, 1996. 
Mervaud, Christine. Voltaire à table : plaisir du corps, plaisir de l'esprit. Paris : Desjonquères, 1998.

Moulin, Léo. Les liturgies de la table : une histoire culturelle du manger et du boire. Paris : Albin Michel, 1989.

Nogler, Tara Lee. Food / Body as Text : Exploring the Cultural / Corporeal Politics of Power. Thèse de maîtrise, Université McMaster, 2000.

Ory, Pascal. Le discours gastronomique français. Paris : Gallimard-Julliard, 1998.

Oxford Symposium on Food. Proceedings ed. by Harlan Walker. For a complete list of past proceedings since 1981: http://www.oxfordsymposium.org.uk/index.html

Parisot, Fabrice. "Les contextes culinaires dans l'œuvre romanesque d'Alejo Carpentier." Nourritures et écriture. Tome 1. Ed. Marie-Hélène Cotoni. U de Nice-Sophia Antipolis, 1999. 233-251.

Paterson, Janet M. Figures de l'autre dans le roman québécois. Québec : Nota Bene, 2004.

Pelchat, Marcia Levin et Fritz Blank. "A Scientific Approach to Flavours and Olfactory Memory." Food and the Memory : Proceedings of the Oxford Symposium on Food and Cookery 2000. Ed. Harlan Walker. Devon, England : Cromwell/Prospect, 2001. 185-191.

Proust, Marcel. Du côté de chez Swann. Paris : Gallimard, 1987.

Rouyer, Marie-Claire, Dir. Food for Thought, ou Les avatars de la nourriture. Groupe d'Études et de Recherches Britanniques, U Michel de Montaigne-Bordeaux. Bordeaux : PU de Bordeaux, 1998.

Schab, Frank R. "Odors and the Remembrance of Things Past." Journal of Experimental Learning, Memory and Cognition 16.4 (1990) : 648-655. 
Schofield, Mary Anne. Cooking by the Boo k: Food in Literature and Culture. Bowling Green, Ohio: Bowling Green U Poplar P, 1989.

Serres, Michel. Les cinq sens : philosophie des corps mêlés. Paris : Grasset, 1985.

Sherzer, Dina. "Violence gastronomique dans Moderato cantabile." French Review 50, 4 (March 1977) : 596-601.

Skubal, Susanne. Word of Mouth : Food and Fiction after Freud. New York : Routledge, 2002.

Sutton, David. Remembrance of Repasts : an Anthropology of Food and Memory. New York: Oxford, 2001.

Tobin, Ronald. Tarte à la crème: Comedy and Gastronomy in Molière's Theater. Columbus : Ohio State UP, 1990.

Toussaint-Samat, Maguelonne. Histoire naturelle et morale de la nourriture. Paris : Bordas, 1987.

Walker, Harlan, Ed. Food and the Memory. Proceedings of the Oxford Symposium on Food and Cookery 2000. Devon, England : Prospect, 2001.

Wilkins, John, Ed. Food in European Literature. Wiltshire, England: Cromwell, 1996.

\footnotetext{
Notes

${ }^{1}$ Qui représente aussi une analyse de type sémiotique.

${ }^{2}$ Par exemple, le sentiment de dégoût du lecteur envers la mendiante pourrait être dû en partie au fait qu'elle chante la bouche pleine. Ainsi, une analyse des manières de table - à l'extérieur du cadre de cette analyse - projetterait de la lumière sur cet aspect relié à la consommation de la nourriture.

${ }^{3}$ Notons que l'histoire de la mendiante cambodgienne est racontée par Peter Morgan, un Anglais. Malgré la distance qui sépare les deux personnages et qui peut influencer la perspective de Peter Morgan sur le "véritable cheminement fictif" de la mendiante, la nourriture que cette dernière ingère et régurgite peut tout de même être analysée en tant que signe culinaire mis dans le texte par l'auteure même, soit Duras. Le manque de voix et la vie narrée de la mendiante par un homme blanc pourrait faire l'objet d'une autre analyse.

${ }^{4}$ Par déchéance, je précise que c'est «l'état d'une personne qui est déchue - (la déchéance est) abaissement, chute, décadence, déclin. » (Petit Robert 638)

${ }_{6}^{5}$ Cette fonction est l'une des fonctions de la nourriture identifiée par Biasin.

${ }^{6}$ Pour plus de détails sur l'échec de la maternité dans LVC, consulter l'article de Mieke Bal (10-11) et celui de Nina Gilbert (322).

7 "Dis-moi ce que tu manges, je te dirai qui tu es" (37), maxime célèbre de Brillat-Savarin.

${ }^{8}$ Le mot "souillure", au départ expliqué par Mary Douglas (Implicit Meanings - Chap.7, Pollution 106-115), a été repris par Kristeva.
} 
${ }^{9}$ Cette transformation finale de la mendiante en être mi-animal, mi-humain rejoint enfin ce que Sherzer préconisait plus tôt (Discourse 372).

${ }^{10}$ Notons que le coût associé à ces denrées est déjà élevé dans le pays d'origine. Leur disponibilité dans un pays aussi éloigné de la France que l'Inde ne fait qu'accroître leur prix, leur rareté - et leur valeur sémiotique.

${ }^{11}$ On pense à L'Aquarium de Godbout. Le comportement d'alcooliques des colonisateurs (excès de consommation) sert d'indicateur de leur corruption morale et de l'altérité ; «ils vivent dans un autre monde ».

${ }^{12} \mathrm{D}$ 'autres contrastes ont été relevés par Sherzer « leisure and work, [...] human and animal, refined and uncouth, named and nameless » (377) et par Bal, silence et communication (13)

${ }^{13}$ Les aliments pourris qu'elle ingère lui ont fait perdre les cheveux, et son pied pourrit pendant les vingt années de narration de l'œuvre. 